\title{
Revision of thickness design of frozen walls considering frost heave
}

\author{
Zhou Xiao $\min ^{1, a}$, Guan Hua-dong ${ }^{1, b}$ and Xie Yan-ke ${ }^{1, c}$ \\ ${ }^{1}$ Full address of first author, including country
}

${ }^{1}$ State Key Laboratory of High - Efficient Mining and Safety of Metal Mines, Ministry of Education, University of Science and Technology Beijing. No.30,Xueyuan Rd, Haidian, Beijing 100083, China

1,austb_zhou@163.com, ${ }^{1, b}$ yidixuezi@126.com, ${ }^{1, c}$ xieyanke1990@163.com

Keywords: Artificial ground freezing, Frost heave, frozen wall design.

\begin{abstract}
The paper outlines development of the thickness design of cylindrical frozen walls in artificial ground freezing (AFG). A plain strain mechanical model coupled with infinite surrounding soil and rock takes into account the frost heave ratio to investigate the influence of frost heave on the thickness design of frozen wall, and superposition method is used to solve the complicated problem of frozen wall swelling. A revised formula referred to as "Baoshen" formula has been proposed. This formula provides a convenient analytic solution for any AGF problem involving not only frost heave but also the action of surrounding soil.
\end{abstract}

\section{Introduction}

Artificial ground freezing (AGF) technology has been widely used in shaft sinking. There has been more than 1,000 shafts successfully sunk by AFG [1]. The thickness design formula has developed significantly over the time. In the early age (1910 to 1950) the shaft sink is very shallow (the depth is less than $100 \mathrm{~m}$ ), the Lame formula, based on plain strain elastic model of cylindrical frozen wall, was used for design. Later, as the depth becomes deeper and deeper, the Lame formula was found to be too conservative. Various formulae have been developed based on partially elastic and partially plastic [2], fully plastic, or fully viscous behavior of the frozen wall. Examples include Domke formula in 1915 and Klein formula in 1968, etc. In 2009, Zhou [3, 4] proposed a new formula based on plain strain elastic model considering the effect of the infinite surrounding rock or soils, which can be used for the design of both shaft lining and frozen wall, especially for shaft sinks built in rocky aquifer

Despite these improvements, there are still many occurences of freeze tube breakage observed in field applications. Many attribute these failures to over-deformation of the ie wall, while others think the strength of the tube material is too low or the joints of freeze tube are too weak.

Frost heave has not been widely considered in the past. Since good performance has been achieved by prolonged deep freezing, we think frost heave can be a very important factor that needs to be considered in artificial ground freezing. In this paper, we present a plain strain model of cylindrical frozen wall enclosed in infinite rock or soil considering frost heave ratio.

Please keep a second copy of your manuscript in your office. When receiving the paper, we assume that the corresponding authors grant us the copyright to use the paper for the book or journal in question. Should authors use tables or figures from other Publications, they must ask the corresponding publishers to grant them the right to publish this material in their paper.

\section{Mechanical principal of AGF of deep shaft}

Before the shaft is excavated and lined, a circular configuration of freeze tubes with a designed spacing is installed in the ground, then chilled brine circulation system are formed, and ground freezing can be achieved if the cooling system is allowed sufficient time to work. After the development of frozen wall meets the requirements for the ground strress support and protection, shaft sinking can be carried outstep by step. Such a procedure of shaft sinking with AGF is illustrated with Fig.1. Fig.1-(1) represents the original state of ground stress, Fig.1-(2) describes the 
second state of ground stress distribution with the forming of frozen wall. Fig.1-(3) presents the third state of ground stress distribution when the excavation is carried out without lining.

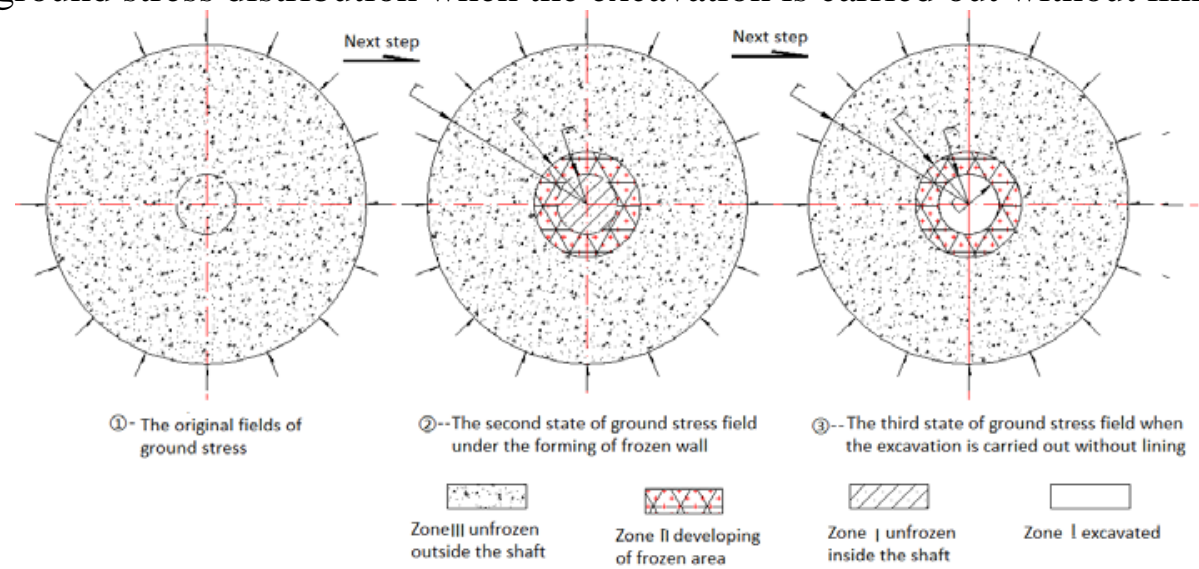

Fig.1 Mechanical Chart of ground freezing and shaft sinking procedure

We aim to analyze the stress distribution at every zone in the third state and to study the safety of the frozen wall. In the second state as described byFig.1-(2), frost heave occurs, and inducesadditional stress to the original stress field. Fig.1-(3) shows the excavation, so the stresses inside the shaft area are released completely, and this causes the stress re-distribution.

Assume the entire procedure is completely elastic, we can apply superposition method to solve the problem as shown in Fig.2.

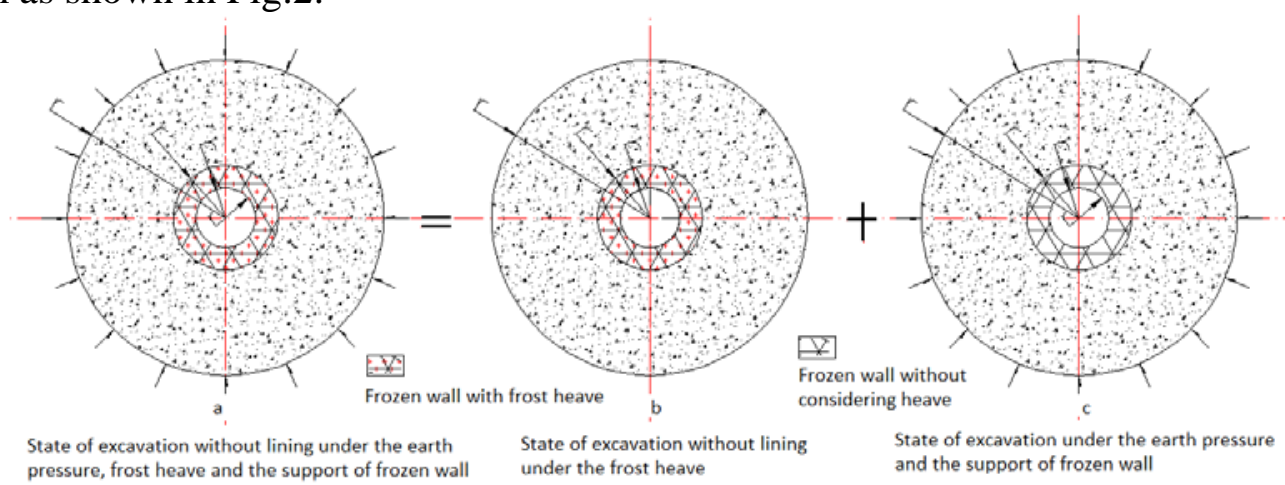

Fig.2 Superposition of mechanics solutions of shaft ground freezing and sinking

Based on the superposition method, the stress field described in Fig.2-a equals to the stress field described in Fig.2-b plus the stress field described in Fig.2-c. we already have the stress field and displacement solutions of Fig.2- c[4] which is the case without consideration of frost heave: Eqn. (1) and (2) are the stress field functions; Eqn.(3) is the displacement field in Area II of frozen wall (refer to Fig. 1 for zone identification).

$$
\begin{gathered}
\sigma_{2 r}^{I_{-}-c}=-\frac{\left(1-\frac{r_{1}^{2}}{r^{2}}\right)}{\left[\frac{G_{1}}{G_{2}}\left(1-2 \mu_{2}\right)+1\right]+\left(\frac{G_{1}}{G_{2}}-1\right) \frac{\mathrm{r}_{1}^{2}}{\mathrm{r}_{2}^{2}}} p_{\infty} \\
\sigma_{2 \theta}^{I I-c}=-\frac{\left(1+\frac{r_{1}^{2}}{r^{2}}\right)}{\left[\frac{G_{1}}{G_{2}}\left(1-2 \mu_{2}\right)+1\right]+\left(\frac{G_{1}}{G_{2}}-1\right) \frac{\mathrm{r}_{1}^{2}}{\mathrm{r}_{2}^{2}}} p_{\infty} \\
u_{f}^{I I-c}(r)=-\frac{r p_{\infty}}{2 G_{2}} \frac{\frac{\mathrm{r}_{1}^{2}}{r^{2}}+\left(1-2 \mu_{2}\right)}{\frac{G_{1}}{G_{2}}\left(1-2 \mu_{2}\right)+1+\left(\frac{G_{1}}{G_{2}}-1\right) \frac{\mathrm{r}_{1}^{2}}{\mathrm{r}_{2}{ }^{2}}}
\end{gathered}
$$

In the next step we aim to find the solution of Fig.2- b. 


\section{Solution}

To solve the problem of Fig.2- b, we divide the model into two parts, and apply the rotational symmetry example of polar coordinate based on elastic theory [5].

Frost heave is produced by the phase change of pore water into pore ice; the pore water commonly exists in various types of soils or rocks, and is affected by the movement of moisture and heat transfer in frozen and unfrozen soil; the frost heave ratio $\delta$ is a function of pressure, temperature gradient and ice penetration rate, time, and so on [6]. Takashi, et al proposed an empirical formula for frost heave ratio:

$$
\delta=\frac{\Delta h}{h}=\delta_{0}+\frac{\sigma_{0}}{\sigma}\left(1+\sqrt{\frac{U_{0}}{U}}\right)
$$

Where $\delta$ is frost heave ratio [no dimensional], $\Delta h$ is the total heave height, $h$ initial soil specimen height, $\sigma$ effective stress which acts parallel to heat flow [MPa], $U$ freezing speed of soil[mm/day], $\delta_{0}, \sigma_{0}, U_{0}$ are the characteristic constants of the soil. In elastic plain analysis of polar coordinates, we can take the frost heave as a strain for constitutive relationship ${ }^{[7]}$ :

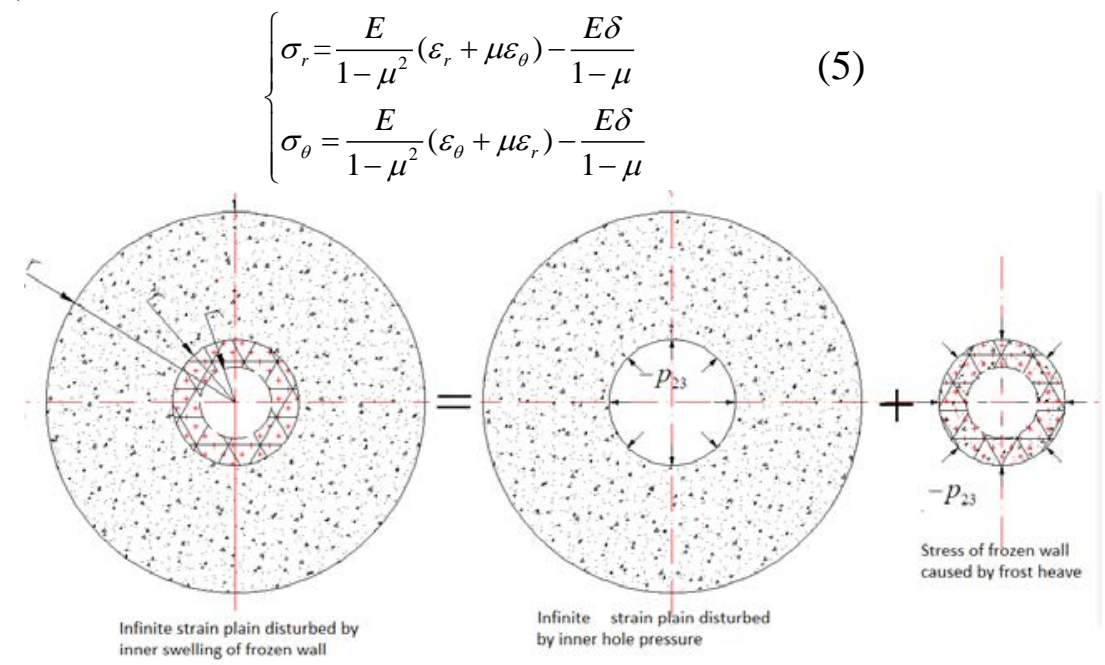

Thus the stress and displacement fields of the frozen wall are as follows:

$$
\begin{aligned}
& \sigma_{r}^{I I}=\frac{2 G_{u}\left(1-\frac{r_{1}^{2}}{r^{2}}\right)}{\left[\frac{G_{u}}{G_{f}}\left(1-2 \mu_{f}\right)+1\right]+\left(\frac{G_{u}}{G_{f}}-1\right) \frac{r_{1}^{2}}{r_{2}^{2}}}\left(1+\mu_{f}\right) \delta \\
& \sigma_{\theta}^{I I}=\frac{2 G_{u}\left(1-2 \mu_{f}\right)\left(1+\frac{r_{1}^{2}}{r^{2}}\right)}{\left[\frac{G_{u}}{G_{f}}\left(1-2 \mu_{2}\right)+1\right]+\left(\frac{G_{u}}{G_{f}}-1\right) \frac{r_{1}^{2}}{r_{2}^{2}}}\left(1+\mu_{f}\right) \delta \\
& u_{2}^{I I}(r)=-\frac{\frac{G_{1}}{G_{2}}\left(\frac{r_{1}^{2}}{r_{2}^{2}}+\frac{r_{1}^{2}}{r^{2}}\right)+1-\frac{r_{1}^{2}}{r_{2}^{2}}}{\left[\frac{G_{1}}{G_{2}}\left(1-2 \mu_{2}\right)+1\right]+\left(\frac{G_{1}}{G_{2}}-1\right) \frac{r_{1}^{2}}{r_{2}^{2}}}\left(1+\mu_{2}\right) \delta r
\end{aligned}
$$

\section{Final solution}

With the solutions of Fig.2 b and Fig.2 c, we combine them based on superposition method to obtain the following stress and displacement field solutions.

$$
\sigma_{r}^{I I}=\frac{\left(2 G_{2} \delta-\frac{G_{2}}{G_{1}} p_{\infty}\right)\left(1-\frac{r_{1}^{2}}{r^{2}}\right)}{\left(\frac{G_{2}}{G_{1}}+1-2 \mu_{2}\right)-\left(\frac{G_{2}}{G_{1}}-1\right) \frac{r_{1}^{2}}{r_{2}^{2}}}
$$




$$
\sigma_{\theta}^{I I}=\frac{\left(2 G_{2} \delta-\frac{G_{2}}{G_{1}} p_{\infty}\right)\left(1+\frac{\mathrm{r}_{1}^{2}}{r^{2}}\right)}{\left(\frac{G_{2}}{G_{1}}+1-2 \mu_{2}\right)-\left(\frac{G_{2}}{G_{1}}-1\right) \frac{r_{1}^{2}}{r_{2}^{2}}}
$$

From Eqn. 10, when $r=r_{1}$, the hoop stress reaches the maximum value as the first principal stress at the inner surface of frozen wall:

$$
\left.\sigma_{\theta}^{I I}\right|_{\max }=\frac{2\left(2 G_{2} \delta-\frac{G_{2}}{G_{1}} p_{\infty}\right)}{\left(\frac{G_{2}}{G_{1}}+1-2 \mu_{2}\right)-\left(\frac{G_{2}}{G_{1}}-1\right) \frac{r_{1}^{2}}{r_{2}^{2}}}
$$

According to third strength theory of material mechanics that is the maximum shearing stress theory of failure, $\left|\sigma_{\theta}^{I I}-\sigma_{r}^{I I}\right|=\left|\sigma_{\theta}^{I I}\right| \leq[\sigma]$, the following formula for frozen wall deign are obtained.

$$
\begin{aligned}
t h= & r_{2}-r_{1}=r_{1}\left(\sqrt{\frac{\alpha[\sigma]}{\beta[\sigma]+4 G_{u} \delta-2 p_{\infty}}}-1\right) \\
& \text { Where: } \alpha=\left(1-\frac{G_{u}}{G_{f}}\right) ; \beta=\left[1+\frac{G_{u}}{G_{f}}\left(1-2 \mu_{f}\right)\right]
\end{aligned}
$$

Eqn.12 is very similar to "Baoshen" formula, with an additional item in the denominator, $4 G_{u} \delta$, which reflect the action of frost heave and shearing modulus. This formula for frozen wall thickness can be called a revised "Baoshen" formula.

\section{Conclusion}

Frost heave is one of the most important factors in artificial ground freezing and yet it has rarely been paid attendion in the past studies. This paper proposes a revised "Baoshen" formula for the design of frozen wall thickness and it for the first time takes into account the impact offrost heave and the surrounding soil enclosing the frozen wall. Further experimental is needed to validate this formula.

\section{Reference}

[1] Zhang wen, China new achievement and situation of shaft sinking with AFG, Shaft sinking Technology, No.3, Vol.33, July, 2012, 4-13.

[2] J.S Harris Ground Freezing in Practice, Published by Thomas Telford Service L.td, Thomas Telford House, I Heron Quay, London E14 4JD, ISBN: 0727719955.

[3] Zhou Xiao-min, Chen Jian-hua, Luo Xiao-qing. Research on the preliminary thickness design of shaft lining in porous rock aquifer [J] .Journal of China Coal Society, 2009,3, 4( 9) : 1 174-1 178 .

[4] Zhou Xiao-min, He Zheng-ping, Ji Hong-guang, Design method of freezing rock wall under high water pressure.

[5] Xu Zhi-lun Elastic mechanics [M], Beijing: Renming Eduation publish house, 2nd edition, Apri.1982.

[6] Xu Xue-zu, Wang Jia-chen, Zhang LI-xin, Physics of frozen soil. Beijing: Science Publish House, 2001, ISBN 7-03-006555-7.

[7] Y.Miyata, S Akagawa, Factors governing s frost heave ratio, Ground Freezing 91. Proceedings of the sixth international symposium on ground freezing Beijing, 10-12, Sep. 1992, P55-64. 